\title{
The non-pathogenic Escherichia coli strain W secretes SsIE via the virulence-associated type II secretion system beta
}

\author{
Mark S DeCanio ${ }^{1,2}$, Robert Landick ${ }^{1,2,3}$ and Rembrandt J F Haft ${ }^{*}$
}

\begin{abstract}
Background: Many pathogenic E. coli strains secrete virulence factors using type II secretory systems, homologs of which are widespread in Gram-negative bacteria. Recently, the enteropathogenic Escherichia coli strain E2348/69 was shown to secrete and surface-anchor SsIE, a biofilm-promoting virulence factor, via a type II secretion system. Genes encoding SsIE and its associated secretion system are conserved in some non-pathogenic E. coli, including the commonly-used W (Waksman) strain.

Results: We report here that E. coli W uses its type II secretion system to export a cognate SsIE protein. SsIE secretion is temperature- and nutrient-dependent, being robust at $37^{\circ} \mathrm{C}$ in rich medium but strongly repressed by lower temperatures or nutrient limitation. Fusing either of two glycosyl hydrolases to the C-terminus of SslE prevented it from being secreted or surface-exposed. We screened mutations that inactivated the type II secretion system for stress-related phenotypes and found that inactivation of the secretion system conferred a modest increase in tolerance to high concentrations of urea. Additionally, we note that the genes encoding this secretion system are present at a hypervariable locus and have been independently lost or gained in different lineages of $E$. coli.

Conclusions: The non-pathogenic E. coli W strain shares the extracellular virulence factor SsIE, and its associated secretory system, with pathogenic E. coli strains. The pattern of regulation of SsIE secretion we observed suggests that SsIE plays a role in colonization of mammalian hosts by non-pathogenic as well as pathogenic E. coli. Our work provides a non-pathogenic model system for the study of SsIE secretion, and informs future research into the function of SsIE during host colonization.
\end{abstract}

Keywords: Type II secretion, Surface display, Escherichia coli, Colonization factor

\section{Background}

Gram-negative bacteria use diverse type II secretion systems (T2SS) to deliver a wide variety of proteins into the extracellular milieu [1,2]. Transport is effected by a membrane-spanning complex of 12-15 structural proteins, generically termed Gsp proteins (for general secretory pathway). Secreted substrates first cross the inner membrane by the Sec or Tat pathways; the Gsp proteins then recognize substrates and transport them across the outer membrane. T2SS function requires several proteins that have homologs in type IV pilus biogenesis systems, including an oligomerized secretin, a

\footnotetext{
* Correspondence: rhaft@wisc.edu

${ }^{1}$ Great Lakes Bioenergy Research Center, University of Wisconsin-Madison, Madison, WI, USA

Full list of author information is available at the end of the article
}

helical protein filament called the pseudopilus, and a prepilin peptidase essential for pseudopilus assembly $[3,4]$.

Secreted proteins serve many purposes, from electron transport to nutrient acquisition, and some are important pathogenicity factors for plant and animal pathogens in the Enterobacteraceae [5,6]. Type II secretion has been extensively studied in pathogenic strains of Escherichia coli, which collectively are known to use two distinct disease-promoting T2SS: the StcE secreting system encoded by the pO157 virulence plasmid [7], and the heat-labile enterotoxin (LT) secreting system common to many pathogenic strains [8]. Recently the latter T2SS was shown for the first time to additionally secrete a non-LT protein, known as SslE, from the enteropathogenic strain E2348/69, thereby promoting biofilm maturation and rabbit colonization by E2348/69 $[9,10]$. The 
sslE gene sits immediately upstream of the T2SSencoding secretory genes, and transcription of $s s l E$ and the gsp genes was shown to be co-regulated in E. coli strain H10407 [11]. In E2348/69, SslE exists as a lipidanchored, surface-exposed protein in the outer membrane and is also released into the culture supernatant. Strozen et al. termed the LT- and SslE-secreting system $\mathrm{T}_{2 \mathrm{SS}} \mathrm{S}_{\beta}$, to distinguish it from the chitinase-secreting $\mathrm{T}^{2 S S_{\alpha}}$ that co-occurs in several E. coli strains [12]. Based on phylogenetic and structural analyses, Dunstan et al. recently determined that the E. coli $\mathrm{T}_{2} \mathrm{SS}_{\beta}$ is part of a larger group of T2SS that contain "Vibrio-type secretins", making it a model for numerous type II secretion systems used to deliver toxic substrates by Vibrio and Escherichia species [10].

The SslE-secreting $\mathrm{T}_{2} \mathrm{SS}_{\beta}$, unlike the StcE-secreting pO157 T2SS, is conserved in several non-pathogenic "safe" strains of E. coli ("safe" strains may colonize hosts, but have never been known to cause disease), including wild-type $\mathrm{B}$ and $\mathrm{W}$ isolates [13]. To date, however, no report has described secretion of proteins by $\mathrm{T}_{2} \mathrm{SS}_{\beta}$ in any non-pathogenic strain. We were interested to determine whether non-pathogenic E. coli could also secrete the "virulence factor" SslE. Secretion of SslE by a safe strain would imply that SslE itself is not capable of promoting a disease state, and would invite comparisons of SslE function between pathogens and non-pathogens. Furthermore, if non-pathogenic E. coli could secrete SslE, the $\mathrm{T}_{2} \mathrm{SS}_{\beta}$ system could be studied using a nonpathogenic model organism.

We demonstrate here that the non-pathogenic E. coli strain $\mathrm{W}$ encodes a functional $\mathrm{T}_{2} \mathrm{SS}_{\beta}$ that secretes a cognate SslE protein. We found a strong effect of growth conditions on SslE secretion, which is relatively robust in rich medium at $37^{\circ} \mathrm{C}$ and undetectable when cells are cultured at $30^{\circ} \mathrm{C}$ or in minimal medium. Previous work suggested that the C-terminus of SslE might be a permissive site for sequence insertions with regards to $\mathrm{T}_{2} \mathrm{SS}_{\beta}$ recognition [9], but we found that C-terminal enzyme fusions to SslE blocked protein secretion and surface display.

As noted above, the $\mathrm{T}_{2} \mathrm{SS}_{\beta}$ was shown to promote mature biofilm formation in E. coli E2348/69. We searched for additional phenotypes in $E$. coli $\mathrm{W}$ by phenotypic microarray analysis of a mutant lacking $\mathrm{T}_{2} \mathrm{SS}_{\beta}$-encoding genes on Biolog stress plates. The phenotypic microarray indicated a potential fitness effect of the mutation in high concentrations of urea. Using standard culture techniques, we found that deletion of $\mathrm{T}_{2} \mathrm{SS}_{\beta}$-encoding genes, or the sslE gene, conferred a small survival advantage in medium containing high concentrations of urea.

Our findings make $\mathrm{T}_{2} \mathrm{SS}_{\beta}$ the only virulence-associated T2SS with shared functions in pathogenic and nonpathogenic E. coli. Considering our regulatory data and the clear homology between the $\mathrm{T}_{2} \mathrm{SS}_{\beta}$-encoding operons of W and E2348/69, we propose that SslE is used by nonpathogenic as well as pathogenic strains of $E$. coli during host colonization.

\section{Results}

\section{E. coli $W$ secretes SsIE using $T 2 S S_{\beta}$ under specific temperature and nutrient conditions}

Prior to publication of the finished E. coli W genome sequence [13], a draft $E$. coli W genomic sequence generated by the U.S. Department of Energy Joint Genome Institute in collaboration with the Great Lakes Bioenergy Research Center (GenBank accession NZ_AEDF00000000) revealed the presence of the entire $\mathrm{T}_{2} \mathrm{SS}_{\beta}$ gene cluster, including a copy of the gene encoding SslE (see Figure 1 for a depiction of the locus). To determine whether E. coli W secreted endogenous SslE via $\mathrm{T}_{2} \mathrm{SS}_{\beta}$, we analyzed the proteomes of the wild-type strain (WT) and a mutant lacking the genes encoding the conserved structural proteins of $22 S_{\beta}(\Delta g s p C-M)$. We grew strains in liquid culture, then harvested cells by centrifugation and compared the proteins present in cell lysates and cell-free supernatants (the latter containing any secreted proteins) by SDSPAGE. We observed a $\sim 180 \mathrm{kDa}$ protein, the expected size for SslE, that was present in the supernatants of WT cultures but not $\Delta g s p$ cultures (Figure 2A). The $\sim 180 \mathrm{kDa}$ protein band was absent from supernatants and cell extracts of a $\Delta s s l E$ strain, but reappeared when we complemented the $s s l E$ deletion with plasmid-encoded $s s l E$ (Figure 2B). To further confirm that SslE was secreted and did not play an intracellular role in activating protein secretion, we attempted to complement the $\Delta s s l E$ strain with a form of SslE lacking the Sec signal peptide (SslE-SP). Unlike wild-type SslE, SslE-SP could not complement the secretory defect in the $\Delta s s l E$ strain. Taken together, our data demonstrate that SslE is secreted from wild-type E. coli $\mathrm{W}$ by $\mathrm{T}^{2} \mathrm{SS}_{\beta}$.

Intracellular SslE did not appear abundant in wild-type E. coli W, even under conditions where secretion of SslE was detectable. We observed accumulation of SslE in the cell when SslE was expressed from a multicopy plasmid, however. We postulate that in wild-type cells, the intracellular concentration of SsIE is maintained at a relatively low level, and that SslE release from cells over time results in accumulation in the supernatant.

Type II secretion systems require prepilin peptidases to produce the mature, functional forms of their prepilin proteins [1], and the prepilin peptidase PppA is required for secretion of LT by $\mathrm{T}^{2 S S_{\beta}}$ in E. coli H10407 [12]. To determine whether PppA is similarly required for SslE secretion by $E$. coli W, we compared SslE secretion in WT to a $\triangle p p p A$ strain. SslE secretion was not detectable in the $\triangle p p p A$ background, and the mutation could be complemented by plasmid-encoded PppA (Figure 2C). 


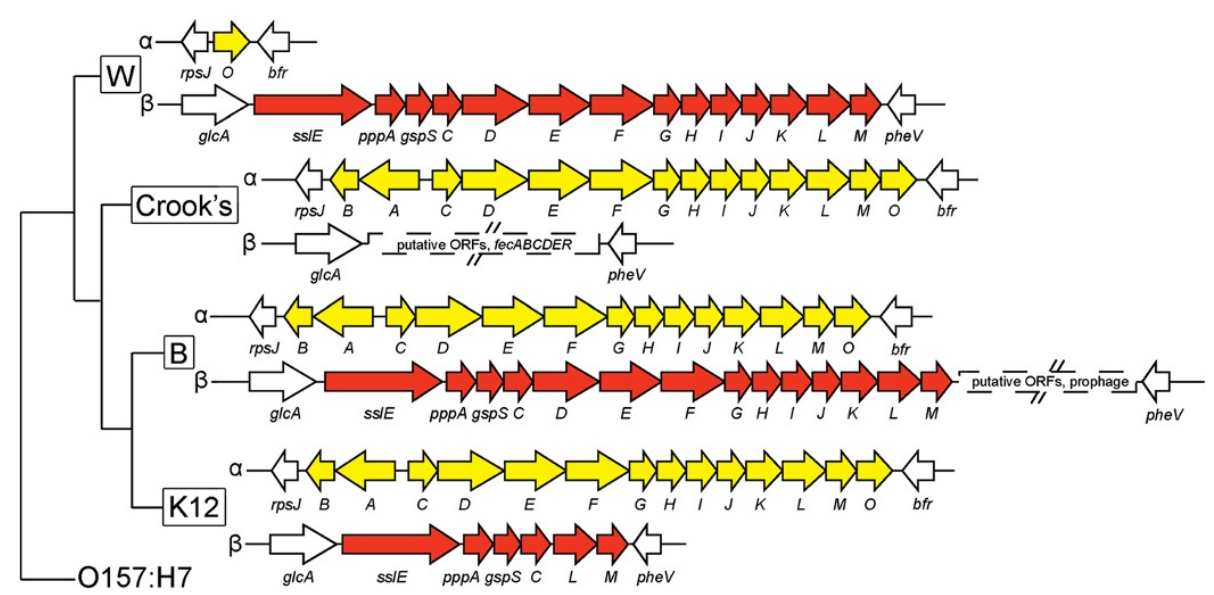

Figure 1 Distribution of $\mathrm{T}_{2} \mathrm{SS}_{\boldsymbol{\alpha}}$ and $\mathrm{T}_{2} \mathrm{SS}_{\boldsymbol{\beta}}$ in non-pathogenic E. coli strains. Phylogeny is from Archer et al. [13], with O157:H7 as an outgroup lacking both $\mathrm{T}_{2} \mathrm{SS}_{\alpha}$ and $\mathrm{T} 2 \mathrm{SS}_{\beta}$. Loci encoding the two T2SS types (where present) are diagrammed for each strain. Branch lengths are arbitrary. $\mathrm{T}_{2} \mathrm{SS}_{\alpha}$ gsp genes are colored yellow, and $\mathrm{T}_{2} \mathrm{SS}_{\beta}$ gsp genes are shown in red.

These results confirm that a fully-functional $\mathrm{T}_{2} \mathrm{SS}_{\beta}$ is required to secrete SslE, and indicate that expression of the $g s p C-M$ genes alone is not sufficient to allow SslE secretion.

We hypothesized that SslE secretion in E. coli W might play a role in host colonization, and that secretion might be regulated such that more SslE is secreted under conditions that resemble the mammalian gut. We assessed this conditionality by examining SslE secretion from cultures grown at different temperatures and nutrient conditions: $30^{\circ} \mathrm{C}$ vs. $37^{\circ} \mathrm{C}$, and minimal MOPSglycerol broth vs. rich LB (Figure 2D). We observed secretion of SslE only in cultures grown in $\mathrm{LB}$ at $37^{\circ} \mathrm{C}$, indicating that either reduced temperature or nutrient limitations are sufficient to block SslE secretion.

\section{C-terminal fusions to SsIE prevent secretion}

In their initial characterization of SslE surface display and secretion, Baldi et al. found that C-terminal fusion of a small tetracysteine-containing motif to SslE did not interfere with localization of SsIE [9]. This result suggested that the C-terminus of SslE might not be important for the recognition of SslE by $\mathrm{T}_{2} \mathrm{SS}_{\beta}$, and thus might be a permissive site for polypeptide fusions. We were interested in testing C-terminal permissiveness for two reasons: first, because it might provide information about the targeting of SslE for secretion (as there are no defined secretory signals for type II secretion substrates), and second, because SslE fusions might be useful to anchor other proteins to the cell surface. We therefore independently fused two plant cell wall degrading enzymes, Cel45A and Pel10A from Cellvibrio japonicus, to the C-terminus of E. coli W SslE and assessed the capacity of these fusion proteins to be secreted or displayed on the cell surface. Both fusions resulted in stable, enzymatically active proteins when expressed in E. coli W. We did not generate fusions to the potentially lipidated N-terminus of SslE to avoid changes in lipidation that could affect protein localization.

We performed all secretion and display experiments side-by-side in wild-type and T2SS-deficient $\triangle p p p A$ strains, and present the results in Table 1. By following activity of the enzymatic fusions, we found that neither fusion protein was released into the medium under conditions in which we found wild-type SslE to be released. Indeed, extracellular activity of SslE-Cel45A was difficult to detect, though lysed cells released highly active enzyme. Because the substrates for Cel45A (carboxymethyl cellulose) and Pel10A (polygalacturonic acid) are high molecular weight polysaccharides that cannot enter the E. coli cell, we were able to assess surface display of fusion proteins by measuring the enzymatic activity of intact cells as compared to cell lysates. These experiments further demonstrated that the fusion proteins were not displayed on the surface of the cell, but accumulated intracellularly.

\section{Inactivation of $\mathrm{T}_{2} \mathrm{SS}_{\beta}$ modestly increases urea tolerance}

Baldi et al. demonstrated that inactivation of $\mathrm{T}^{2} \mathrm{SS}_{\beta}$ in E. coli E2348/69 inhibited biofilm maturation in confocal microscopic analysis of flow cell cultures, though it had no effect on early biofilm development in stationary plate assays [9]. To uncover other phenotypes related to $\mathrm{T}_{2} \mathrm{SS}_{\beta}$ disruption, we used $E$. coli $\mathrm{W}$ as a non-pathogenic model system in a partial Biolog phenotypic microarray to compare wild-type and $\Delta g s p$ strains grown with various stressors. The Biolog dye-reduction traces are presented in Additional file 1. Under most conditions the two strains were indistinguishable, but the screen indicated that elevated urea concentrations might differentially affect their 


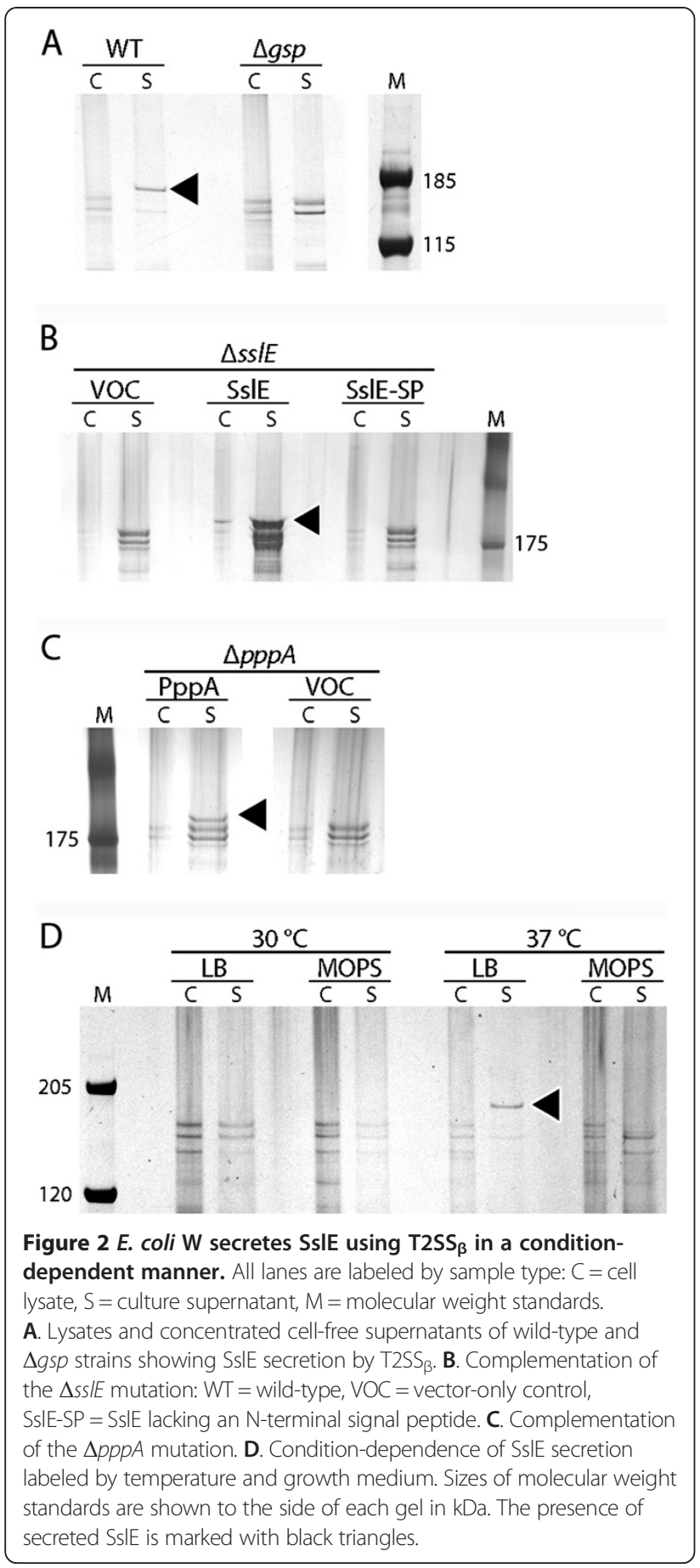

growth. We examined this phenomenon in 96-well plate growth experiments under conditions in which our data showed SslE to be secreted ( $\mathrm{LB}$ at $37^{\circ} \mathrm{C}$ ). Compared to the wild-type control, $\triangle g s p$ and $\triangle p p p A$ strains maintained higher stationary-phase densities in the presence of $0.90 \mathrm{M}$ and $1.15 \mathrm{M}$ urea (Additional file 2: Figure S1), suggesting that inactivation of the $\mathrm{T}_{2} \mathrm{SS}_{\beta}$ system modestly increased urea tolerance even when the structural Gsp proteins were still expressed. We determined the role of
Table 1 Extracellular and surface-displayed activity of SsIE-Cel45A and SsIE-Pel10A from liquid cultures

\begin{tabular}{|c|c|c|c|c|}
\hline \multirow[t]{2}{*}{ Strain } & \multicolumn{2}{|c|}{ SsIE-Cel45A activity ${ }^{a}$} & \multicolumn{2}{|c|}{ SsIE-Pel10A activity ${ }^{\mathrm{a}}$} \\
\hline & $\begin{array}{l}\text { Supernatant } \\
\text { fraction }^{b}\end{array}$ & $\begin{array}{l}\text { Displayed } \\
\text { fraction }^{c}\end{array}$ & $\begin{array}{c}\text { Supernatant } \\
\text { fraction }^{\mathrm{b}}\end{array}$ & $\begin{array}{c}\text { Displayed } \\
\text { fractionc }^{c}\end{array}$ \\
\hline WT pRH153 & $<0.01$ & $<0.01$ & - & - \\
\hline$\Delta p p p A$ pRH153 & $<0.01$ & $<0.01$ & - & - \\
\hline WT pRH154 & - & - & $0.11(1)$ & $0.08(1)$ \\
\hline$\Delta p p p A \mathrm{pRH} 154$ & - & - & $0.10(4)$ & $0.095(5)$ \\
\hline
\end{tabular}

${ }^{\text {a }}$ Values shown are means of at least two biological replicates, with error in the last digit denoted parenthetically.

b Extracellular activity divided by the activity from an equivalent fraction of lysed culture.

c Activity measured using intact cells divided by the activity from an equivalent fraction of lysed culture.

SslE in this phenotype and verified modest urea tolerance by following the growth and viability of wild-type, $\Delta g s p$, and $\Delta s s l E$ strains for 48 hours with or without $1.15 \mathrm{M}$ urea under the standard culture conditions we used for SslE secretion experiments (in culture tubes on a rolling wheel for vigorous aeration). Culture absorbance readings and viable cell counts indicated that, without urea, the three strains grew equivalently up to 12 hours and slowly lost viability between 12 and 48 hours, with indistinguishable final viable counts at 48 hours (Figure 3 and Table 2). In the presence of $1.15 \mathrm{M}$ urea all strains grew poorly, but $\triangle g s p$ and $\triangle s s l E$ strains maintained higher turbidity and viable cell counts than wild-type, with both mutants having $>60 \%$ more surviving cells than wild-type at 48 hours. We conclude that the inability to secrete SslE confers a small survival advantage in the presence of high concentrations of urea.

\section{Discussion and conclusions}

Strains within the species Escherichia coli encode different combinations of type II secretion systems, each of which secrete different effectors and presumably provide specific advantageous phenotypes to their host organisms. To this point, the only T2SS shown to be functional in non-pathogenic $E$. coli strains is the chitinase-secreting $\mathrm{T}_{2} \mathrm{SS}_{\alpha}$, which is the sole T2SS encoded by E. coli K-12 $[13,14]$ and whose role in natural environments is unknown. We demonstrate here that, surprisingly, the $\mathrm{T}^{2} \mathrm{SS}_{\beta}$ that promotes virulence of the enterotoxic strain $\mathrm{H} 10407$ and the enteropathogenic strain E2348/69 is conserved, and secretes a virulence factor homolog, in the nonpathogenic E. coli W strain. To our knowledge, this is the first time a virulence-associated type II secretion system has been shown to function in non-pathogenic $E$. coli. Deletion of $s s l E$ could be complemented in trans, indicating that an $s S l E$ disruption does not prevent expression or assembly of $\mathrm{T}^{2 S S_{\beta}}$ in $E$. coli W. We observed that $E$. coli W preferentially secretes SslE under nutrient-rich conditions at human body temperature $\left(37^{\circ} \mathrm{C}\right)$, which suggests that 


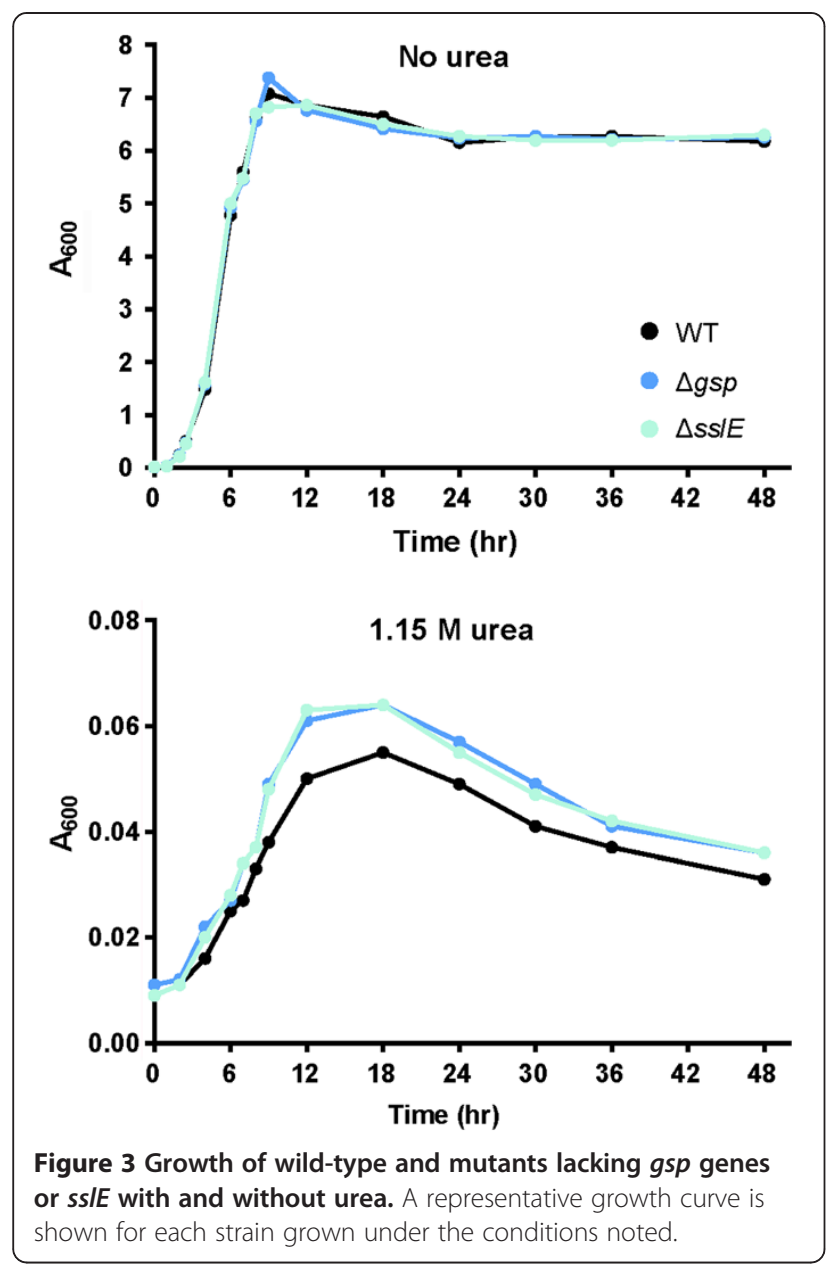

SslE may be a colonization factor in non-pathogenic strains. The regulation of SslE secretion in other strains is unclear, but expression of genes encoding the LT-secreting $\mathrm{T}_{2} \mathrm{SS}_{\beta}$ in E. coli $\mathrm{H} 10407$ was also shown to be upregulated at host-associated temperatures [11]. We hope that future experiments will elucidate the role of SslE in host colonization by non-pathogenic E. coli.

If secretion of SslE indeed aids diverse E. coli in gut colonization, it is perhaps surprising that some gutderived isolates of $E$. coli, such as $\mathrm{K}-12$ and $\mathrm{O} 157: \mathrm{H} 7$, lack the T2SS responsible for SslE secretion. Such strains may compensate for the loss of biofilm-forming propensity using other mechanisms; strains bearing the F plasmid (such as wild-type K-12) may rely on F pilus-mediated aggregation [15], for example. The genes encoding the SslEsecreting $\mathrm{T}_{2} \mathrm{SS}_{\beta}$ are present adjacent to the pheV tRNA gene, which appears to be a hypervariable locus in $E$. coli [16-18], so they may be randomly lost at a relatively high rate. Indeed, a comparison between phylogeny and $\mathrm{T}_{2} \mathrm{SS}_{\alpha} /$ $\mathrm{T}^{2} \mathrm{SS}_{\beta}$ presence suggests independent losses of $\mathrm{T}^{2} \mathrm{SS}_{\beta}$ in non-pathogenic strains (Figure 1). Notably, B and W encode the complete $\mathrm{T}_{2} \mathrm{SS}_{\beta}$, while Crook's and $\mathrm{K}-12$ do not, in spite of the fact that Crook's diverged from K-12 prior to the divergence of B. This indicates that either Crook's and $\mathrm{K}-12$ lost the $\mathrm{T}_{2} \mathrm{SS}_{\beta}$-encoding genes independently, or that an ancestor of Crook's, B, and K-12 lost the genes, which were subsequently re-acquired by strain B. An examination of the $\mathrm{T}_{2} \mathrm{SS}_{\beta}$-encoding loci in Crook's and K-12 strongly supports the former explanation. In K-12, the $\mathrm{T}_{2} \mathrm{SS}_{\beta}$-encoding gsp operon clearly experienced an internal deletion that removed the $g s p D-K_{\beta}$ genes, inactivating the T2SS. In Crook's, however, the homologous genomic locus appears entirely different: all $g s p$ genes are absent, and in their place is the fec operon (encoding a ferric citrate transport system) and a variety of putative ORFs. We infer that the most parsimonious explanation of the phylogenetic distribution of $\mathrm{T}^{2} \mathrm{SS}_{\beta}$ is that K-12 and Crook's both lost the T2SS at different points in their evolutionary histories. It remains an open question what pattern of gene gains and losses best explains the distribution of $\mathrm{T}_{2} \mathrm{SS}_{\beta}$ across the diversity of $E$. coli strains not considered in our analysis.

It is of interest to note that a non-polar deletion of the pppA gene, encoding a prepilin peptidase, prevents secretion of SslE by E. coli W. This result agrees with a similar experiment performed by Strozen et al. to assess effects of PppA on LT secretion in H10407 [12]. Both W and $\mathrm{H} 10407$ also encode a second prepilin peptidase (GspO) whose homolog is functional in facilitating ChiA secretion via $\mathrm{T}_{2} \mathrm{SS}_{\alpha}$ in $\mathrm{K}-12$ [19]. Whether the GspO peptidase is not expressed under conditions associated with SslE secretion in both W and H10407, or whether

Table 2 Viable cell counts for cultures grown with and without urea

\begin{tabular}{lccccc}
\hline Strain & Urea $^{\mathbf{a}}$ & $\mathbf{6} \mathbf{h r}^{\mathbf{b}}$ & $\mathbf{1 2} \mathbf{h r}^{\mathbf{b}}$ & $\mathbf{2 4}_{\mathbf{h r}}^{\mathbf{b}}$ & $\mathbf{4 8} \mathbf{h r}^{\mathbf{b}}$ \\
\hline Wild-type & - & $2.8 \pm 0.1 \times 10^{9}$ & $6.9 \pm 0.3 \times 10^{9}$ & $2.0 \pm 0.3 \times 10^{9}$ & $1.2 \pm 0.1 \times 10^{9}$ \\
$\Delta$ gsp & - & $2.6 \pm 0.3 \times 10^{9}$ & $6.2 \pm 0.2 \times 10^{9}$ & $2.4 \pm 0.2 \times 10^{9}$ & $1.2 \pm 0.1 \times 10^{9}$ \\
$\Delta$ ssIE & - & $2.7 \pm 0.1 \times 10^{9}$ & $5.7 \pm 0.2 \times 10^{9}$ & $2.3 \pm 0.3 \times 10^{9}$ & $1.2 \pm 0.1 \times 10^{9}$ \\
Wild-type & + & $5.8 \pm 0.3 \times 10^{6}$ & $3.2 \pm 0.1 \times 10^{6}$ & $1.6 \pm 0.1 \times 10^{6}$ & $3.1 \pm 0.1 \times 10^{5}$ \\
$\Delta$ gsp & + & $7.9 \pm 0.9 \times 10^{6}$ & $4.1 \pm 0.2 \times 10^{6}$ & $2.2 \pm 0.2 \times 10^{6}$ & $5.7 \pm 0.3 \times 10^{5}$ \\
$\Delta$ ssIE & + & $6.3 \pm 0.3 \times 10^{6}$ & $4.1 \pm 0.3 \times 10^{6}$ & $2.1 \pm 0.4 \times 10^{6}$ & $5.0 \pm 0.6 \times 10^{5}$ \\
\hline
\end{tabular}

a - , no urea present; +, 1.15 M urea present.

b Colony-forming units per $\mathrm{ml}$ of culture at the indicated time after inoculation, shown as means \pm SEM for at least three replicate plate counts. 
the two peptidases display different substrate specificities, remains to be determined.

Strikingly, in the presence of the otherwise intact gsp operon, deletion of $s s l E$ was effective in promoting modest urea tolerance. When we first observed the ureatolerant phenotype of the $\Delta g s p$ strain, we hypothesized that the mutant's advantage stemmed from lacking the transmembrane components of the T2SS, particularly the secretin pore in the outer membrane, which might be denatured by urea. The urea tolerance of the $\Delta s s l E$ mutant rules out this hypothesis, however, and indicates that secretion of SslE by $\mathrm{T}_{2} \mathrm{SS}_{\beta}$ renders cells modestly more sensitive to urea. Relative urea sensitivity is likely due to indirect effects on cell physiology of bearing surface-displayed SslE or of releasing of SslE into the culture medium.

We report here that enzymatic fusions to the C-terminus of SslE interfere with its targeting to the T2SS, as measured by release of fusion proteins and by display of fusion proteins on the outer leaflet of the outer membrane. Previously, Baldi et al. fused a tetracysteine motif to the C-terminus of E2348/69 SslE and saw that the fusion protein was still displayed on the cell surface [9]. We do not think these results contradict ours, due to the significant structural differences between the fusion proteins in question. We propose that the six amino acids appended to the C-terminus of SslE in the study by Baldi et al. did not affect secretion of SslE, but that our fusions of SslE to large tightly-folded proteins (plant cell wall degrading enzymes from Cellvibrio japonicus) occluded important targeting motifs recognized by the T2SS. The uncharacterized nature of T2SS recognition of substrates [20] unfortunately limits our ability to speculate further as to what these motifs might be. Future dissection of the SslE protein with internal deletions and protein fusions may yield new insights into the targeting motif(s) of SslE, and determine whether SslE fusions can be used in the surface display of other proteins.

\section{Methods}

\section{Growth media, strains and plasmids}

E. coli strains and plasmids used in this study are summarized in Table 3, and sequences of the plasmids are provided in Additional file 3. The rich (LB) and minimal (Neidhardt MOPS minimal with $0.2 \%$ glycerol) media $[21,22]$ contained supplements at the following concentrations: $25 \mu \mathrm{g} / \mathrm{ml}$ kanamycin, $100 \mu \mathrm{g} / \mathrm{ml}$ ampicillin, and $30 \mu \mathrm{g} / \mathrm{ml}$ chloramphenicol. Mutant strains were constructed by replacing various loci with a FRT-kan-FRT cassette via the $\lambda$ Red method, and kan cassettes were then removed by FLP excision as described $[23,24]$. The FRT-kan-FRT cassette used for gene disruptions of $g s p C$ $M, p p p A$, and $s s l E$ was amplified from Keio mutant genomic DNA [24] using the primer pairs noted in Table 4. To ensure our phenotypes did not result from secondsite mutations, we generated all mutant strains twice in parallel and performed assays with two independent isolates, which behaved similarly in all cases.

All synthetic DNA for plasmid constructions described below was provided by Geneart (Regensburg, Germany).

Table 3 Strains and plasmids used in this study

\begin{tabular}{|c|c|c|}
\hline E. coli strain or plasmid & Description $^{a}$ & Reference or source \\
\hline \multicolumn{3}{|l|}{ Strains } \\
\hline W & Wild-type E. coli W & ATCC 9637 \\
\hline W $\Delta g s p:: K a n$ & W $\Delta g s p C-M:: F R T-k a n-F R T$ & This work \\
\hline W $\Delta g s p:: F R T$ & W $\Delta g s p C-M:: F R T$, derived by FLP recombination from W $\Delta g s p:: K a n$ & This work \\
\hline W $\triangle p p p A:: K a n$ & W $\triangle p p p A:: F R T-k a n-F R T$ & This work \\
\hline W $\triangle p p p A:: F R T$ & W $\triangle p p p A::$ FRT, derived by FLP recombination from W $\Delta p p p A:: K a n$ & This work \\
\hline W $\Delta s s I E:: K a n$ & W $\Delta s s I E:: F R T-k a n-F R T$ & This work \\
\hline W $\Delta s S I E:: F R T$ & W $\Delta$ ssIE::FRT, derived by FLP recombination from W $\Delta$ ssIE::Kan & This work \\
\hline Plasmids & & This work \\
\hline $\mathrm{pRH} 21$ & pACYC184-derived; trc promoter; $\left.||_{a c}\right|^{a}$ & This work \\
\hline $\mathrm{pRH} 31$ & pTrc99A-derived; trc promoter; $\mid$ lac $\left.\right|^{q}$ & This work \\
\hline pMSD6 & $\mathrm{pRH} 21$ with ssIE cloned into the MCS & This work \\
\hline pMSD7 & $\begin{array}{l}\text { pRH21 with ssIE lacking the signal peptide-encoding } \\
\text { sequence cloned into the MCS }\end{array}$ & This work \\
\hline pMSD8 & $\mathrm{pRH} 21$ with pppA cloned into the MCS & This work \\
\hline pRH153 & pRH31 with an ssIE-cel45A fusion cloned into the MCS & This work \\
\hline $\mathrm{pRH} 154$ & pRH31 with an ssIE-pel10A fusion cloned into the MCS & This work \\
\hline
\end{tabular}

${ }^{a}$ MCS, multiple cloning site.

${ }^{b}$ ATCC, American Type Culture Collection. 
Table 4 Primers used in this study

\begin{tabular}{|c|c|c|}
\hline Name & Description & Application \\
\hline gspKO-up & GACAATCTTTAATACAGACAAAGAGCATCTGCGAAAAATTGTACGCGGGATTCCGGGGATCCGTCGACC & gspC-M deletion \\
\hline gspKO-dn & CGCCACGTTAACGAGAGTAATTITATTGATACTAATCTCCTGATACTTTATGTAGGCTGGAGCTGCTTCG & gspC-M deletion \\
\hline pppAKO-dn & ATACACTTGCAGGCCCGCATCCGGCAAGTTACAACAAACAACCTTTAACCATTCCGGGGATCCGTCGACC & pppA deletion \\
\hline pppAKO-dn & TTATTAATAAGAGTTAAAATGTCACTITGATAATGACGTTGTTATCATTATGTAGGCTGGAGCTGCTTCG & pppA deletion \\
\hline ssIEKO-up & TTCTCCCAGTTACGAATTITTAACATTGTTTTGTCACTTGCGTTATTAATTCCGGGGATCCGTCGACC & sslE deletion \\
\hline ssIEKO-dn & TTATTTCATGCCGGATGCGGCGTGAACGCCTTATCCGGCATACAGGATTATGTAGGCTGGAGCTGCTTCG & sslE deletion \\
\hline pppA-up & TTATTAGGTACCATGCTITITGATGTITTCAGC & pppA cloning \\
\hline pppA-dn & ATATTAGGATCCTTAAAACAATGCCTGTAGATAAATTG & pppA cloning \\
\hline sslE-up & TTATTAGGTACCATGAATAAGAAATTTAAATATAAGAAATCG & ssIE cloning \\
\hline sslE-noSP-up & TTATTAGGTACCATGTCTTCCTCCGATACG & SSIE-SP cloning \\
\hline sslE-dn & TTATTAGGATCCTTACTCGACAGACATCTTATG & sslE cloning \\
\hline sslE-dn-nostop & TTATTAGGATCCGCTCTCGACAGACATCTTATG & sslE cloning for fusions \\
\hline cel45A-noSP-up & TTATTAGGATCCGCAGTTTGTGAATATCGTGTTACC & ssIE-cel45A fusion \\
\hline cel45A-dn & TTATTAAAGCTITACGGGCAGGTATTACGAATATC & sslE-cel45A fusion \\
\hline pel10A-noSP-up & TTATTAGGATCCGCCTGCAGTTACAAGGTCAC & sslE-pel10A fusion \\
\hline pel10A-dn & TTATTAAAGCTITTACAGGTAACCCACTTTCTGG & sslE-pel10A fusion \\
\hline
\end{tabular}

Plasmid pRH21 was constructed from pEG100 [25] by replacing the multiple cloning site (MCS) with a synthetic variant including a tandem His-FLAG tag and by adding the $r r n B$-derived terminators from pTrc99A downstream of the MCS. pRH31 was constructed from pTrc99A [26] by replacing the MCS with the same synthetic variant as in pRH21. pMSD6 was constructed using sslE amplified from E. coli $\mathrm{W}$ genomic DNA with the sslE-up and sslE-dn primers (Table 4). pMSD7 was constructed using $s s l E$ similarly amplified with the sslEnoSP-up and sslE-dn primers. pMSD8 was constructed using pppA similarly amplified with the pppA-up and pppA-dn primers. For construction of pMSD6, pMSD7, and pMSD8, the PCR products were digested with Acc65I and BamHI and ligated into the large Acc65I/ BamHI fragment of pRH21.

For construction of pRH153, sslE was amplified from E. coli $\mathrm{W}$ genomic DNA using primers sslE-up and sslEdn-nostop, and the PCR product was digested with Acc65I and BamHI. A gene encoding the mature form of Cel45A from Cellvibrio japonicus Ueda107 was synthesized and codon optimized for E. coli expression, then amplified using the cel45A-noSP-up and cel45A-dn primers, and the PCR product was digested with $\mathrm{BamHI}$ and HindIII. The two digested PCR products (sslE and cel45A) were ligated into the large Acc65I/HindIII fragment of pRH31. pRH154 was constructed as pRH153, with a synthetic gene encoding mature Pel10A from C. japonicus Ueda107 (with altered codon usage for expression in E. coli) being amplified using the pel10AnoSP-up and pel10A-dn primers prior to digestion and ligation.

\section{Protein expression and detection}

For assessing secretion of wild-type SslE, cultures of indicated strains (mutants were all kan-marked, except $\triangle p p p A$ mutants, which were unmarked) were grown in liquid media ( $\mathrm{LB}$ at $37^{\circ} \mathrm{C}$ unless otherwise noted) with aeration for 16-20 hours. For complementation of the $\triangle S s l E$ mutation, gene expression from plasmids was induced with $1 \mu \mathrm{M}$ isopropyl- $\beta$-D-galactopyranoside (IPTG). Cells were harvested by centrifugation and resuspended in SDS sample buffer (SSB) [21] according to the following formula: resuspension volume (in $\mu \mathrm{l})=$ $100 \times \mathrm{A}_{600} \times \mathrm{vol}$ harvested (in $\mathrm{ml}$ ). These concentrated cell lysates were diluted 1:100 in SSB for SDS-PAGE. Cell-free supernatants were concentrated $\sim 10$-fold by filtration using Centricon spin columns (Millipore, Billerica, MA, USA), and added to concentrated SSB for SDS-PAGE. Samples were separated on 4-12\% SDS-polyacrylamide gels and stained with silver to visualize protein bands [21]. SslE secretion experiments were repeated 2-4 times, and single representative gels are shown.

To produce the images in Figure 2, the stained gels were digitally photographed and gel images were enhanced using Adobe Photoshop software. Linear transformations (contrast and brightness adjustments) were applied to the images for clarity; such transformations were applied uniformly across any given gel image.

\section{Fusion protein localization by enzyme activity}

To measure secretion and surface display of SslEenzyme fusions, cultures of WT and $\triangle p p p A:: F R T$ strains bearing the indicated plasmids were grown in $\mathrm{LB}$ at $37^{\circ} \mathrm{C}$ with aeration for 16-20 hours. Cells were harvested by 
centrifugation, and cell-free supernatants were removed; an aliquot of collected cells was removed and lysed using the PopCulture reagent from Novagen (Madison, WI, USA). Enzymatic activities associated with intact cells, lysed cells, and cell-free supernatants were then immediately measured. SslE-Cel45A activity was measured using the CRACC assay [27], and SslE-Pel10A activity was measured using the pectate lyase assay described by Collmer [28].

\section{Growth comparisons}

Phenotypic microarray experiments were performed using an OmniLog reader (Biolog, Hayward, CA, USA) as per the manufacturer's instructions using plate types PM-9 and PM-10. Cultures were grown at $37^{\circ} \mathrm{C}$ for 48 hours, and respiration data were analyzed using the PM software provided with the OmniLog reader. Strains used were wildtype W and $\Delta g s p::$ FRT (unmarked deletion of $g s p C-M$ ).

To compare urea tolerances in 96-well plates, wildtype, $\Delta g s p:: F R T$, and $\triangle p p p A::$ FRT strains were cultured in $200 \mu \mathrm{l}$ aliquots of LB containing $0,0.9 \mathrm{M}$, or $1.15 \mathrm{M}$ urea in 96-well plates (inoculated as 1:100 dilutions from LB overnight cultures). Plates were grown with shaking at $37^{\circ} \mathrm{C}$ in a Tecan M1000 plate reader (Durham, NC, USA). Growth and survival were followed by regular measurement of $\mathrm{A}_{595}$ for each culture.

To compare urea tolerances in glass culture tubes, wildtype, $\Delta g s p:: F R T$, and $\Delta s s l E:: F R T$ strains were cultured in $8 \mathrm{ml}$ volumes of LB containing no urea or $1.15 \mathrm{M}$ urea on a rolling wheel at $37^{\circ} \mathrm{C}$. Biological duplicate cultures of each strain were inoculated with 1:1000 dilutions from LB overnight cultures after verification that all overnight cultures grew to equivalent $\mathrm{A}_{600}$ turbidity readings. Turbidity in growing cultures was measured by reading $\mathrm{A}_{600}$ using a Spectronic 20D digital spectrophotometer; for cultures with high densities $\left(\mathrm{A}_{600}>1.5\right)$, aliquots of the culture were diluted 1:10 or 1:20 prior to measurement of $A_{600}$. Viable cells were enumerated by 10 -fold serial dilution of cultures into sterile $0.9 \% \mathrm{NaCl}$ followed by plating of dilutions on non-selective media and colony counting.

\section{Availability of supporting data}

Biolog cultivation data are included as Additional file 1. Data from microtiter plate growth experiments of cells under urea stress are included as in Additional file 2: Figure S1. The sequences of all plasmids described in this study are included as Additional file 3.

\section{Additional files}

Additional file 1: Dye reduction traces for Biolog experiments.

Additional file 2: Figure S1. Growth of wild-type and mutant strains with and without urea in 96-well plate experiments.

Additional file 3: Sequences of plasmids used in this study.

\section{Abbreviations}

T2SS: Type II secretion systems; LT: Heat-labile enterotoxin; Gsp: General secretory pathway; WT: Wild-type; MCS: Multiple cloning site.

\section{Competing interests}

The authors declare no competing interests.

\section{Authors' contributions}

$M D, R L$, and $R H$ designed experiments and contributed to writing the manuscript. MD and $\mathrm{RH}$ performed experiments and analyzed data. All authors read and approved the final manuscript.

\section{Acknowledgements}

We would like to thank David Keating for thoughtful discussions and critical review of the manuscript. This work was funded by the DOE Great Lakes Bioenergy Research Center (DOE BER Office of Science DE-FC02-07ER64494). Sequencing of E. coli W by the U.S. Department of Energy Joint Genome Institute is supported by the Office of Science of the U.S. Department of Energy under Contract No. DE-AC02-05CH11231.

\section{Author details}

${ }^{1}$ Great Lakes Bioenergy Research Center, University of Wisconsin-Madison, Madison, WI, USA. ²Department of Biochemistry, University of WisconsinMadison, Madison, WI, USA. ${ }^{3}$ Department of Bacteriology, University of Wisconsin-Madison, Madison, WI, USA.

Received: 11 March 2013 Accepted: 4 June 2013

Published: 12 June 2013

\section{References}

1. Korotkov KV, Sandkvist M, Hol WG: The type II secretion system: biogenesis, molecular architecture and mechanism. Nat Rev Microbiol 2012, 10:336-351.

2. McLaughlin LS, Haft RJF, Forest KT: Structural insights into the type II secretion nanomachine. Curr Opin Struct Biol 2012, 22:208-216.

3. Peabody CR, Chung YJ, Yen MR, Vidal-Ingigliardi D, Pugsley AP, Saier MH Jr: Type II protein secretion and its relationship to bacterial type IV pili and archaeal flagella. Microbiology 2003, 149:3051-3072.

4. Hobbs M, Mattick JS: Common components in the assembly of type 4 fimbriae, DNA transfer systems, filamentous phage and protein-secretion apparatus: a general system for the formation of surface-associated protein complexes. Mol Microbiol 1993, 10:233-243.

5. Cianciotto NP: Type II secretion: a protein secretion system for all seasons. Trends Microbiol 2005, 13:581-588.

6. Sandkvist M: Type II secretion and pathogenesis. Infect Immun 2001, 69:3523-3535.

7. Lathem WW, Grys TE, Witowski SE, Torres AG, Kaper JB, Tarr PI, Welch RA: StcE, a metalloprotease secreted by Escherichia coli O157:H7, specifically cleaves C1 esterase inhibitor. Mol Microbiol 2002, 45:277-288.

8. Tauschek M, Gorrell RJ, Strugnell RA, Robins-Browne RM: Identification of a protein secretory pathway for the secretion of heat-labile enterotoxin by an enterotoxigenic strain of Escherichia coli. Proc Natl Acad Sci USA 2002, 99:7066-7071.

9. Baldi DL, Higginson EE, Hocking DM, Praszkier J, Cavaliere R, James CE, Bennett-Wood V, Azzopardi Kl, Turnbull L, Lithgow T, et al: The type II secretion system and its ubiquitous lipoprotein substrate, SsIE, are required for biofilm formation and virulence of enteropathogenic Escherichia coli. Infect Immun 2012, 80:2042-2052.

10. Dunstan RA, Heinz E, Wijeyewickrema LC, Pike RN, Purcell AW, Evans TJ, Praszkier J, Robins-Browne RM, Strugnell RA, Korotkov KV, Lithgow T: Assembly of the type II secretion system such as found in Vibrio cholerae depends on the novel pilotin AspS. PLoS Pathog 2013, 9:e1003117.

11. Yang J, Baldi DL, Tauschek M, Strugnell RA, Robins-Browne RM: Transcriptional regulation of the yghJ-pppA-yghG-gspCDEFGHIJKLM cluster, encoding the type II secretion pathway in enterotoxigenic Escherichia coli. J Bacterio/ 2007, 189:142-150.

12. Strozen $T G$, Li G, Howard SP: YghG $\left(G s p S_{\beta}\right)$ is a novel pilot protein required for localization of the $\mathrm{GspS}_{\beta}$ type II secretion system secretin of enterotoxigenic Escherichia coli. Infect Immun 2012, 80:2608-2622.

13. Archer CT, Kim JF, Jeong H, Park JH, Vickers CE, Lee SY, Nielsen LK: The genome sequence of E. coli W (ATCC 9637): comparative genome 
analysis and an improved genome-scale reconstruction of E. coli. BMC Genomics 2011, 12:9.

14. Blattner FR, Plunkett G, Bloch CA, Perna NT, Burland V, Riley M, ColladoVides J, Glasner JD, Rode CK, Mayhew GF, et al: The complete genome sequence of Escherichia coli K-12. Science 1997, 277:1453-1462.

15. Lawley TD, Wilkins BM, Frost L: Bacterial conjugation in Gram-negative bacteria. In Plasmid biology. Edited by Phillips G, Funnell BE. Washington, D. C: ASM Press; 2004:203-226.

16. Rumer L, Jores J, Kirsch P, Cavignac Y, Zehmke K, Wieler LH: Dissemination of pheU- and pheV-located genomic islands among enteropathogenic (EPEC) and enterohemorrhagic (EHEC) E. coli and their possible role in the horizontal transfer of the locus of enterocyte effacement (LEE). Int $J$ Med Microbiol 2003, 292:463-475.

17. Vimr ER, Steenbergen SM: Mobile contingency locus controlling Escherichia coli K1 polysialic acid capsule acetylation. Mol Microbiol 2006, 60:828-837.

18. Schneider G, Dobrindt U, Bruggemann H, Nagy G, Janke B, Blum-Oehler G, Buchrieser C, Gottschalk G, Emody L, Hacker J: The pathogenicity islandassociated K15 capsule determinant exhibits a novel genetic structure and correlates with virulence in uropathogenic Escherichia coli strain 536. Infect Immun 2004, 72:5993-6001.

19. Francetic O, Pugsley AP: The cryptic general secretory pathway (gsp) operon of Escherichia coli K-12 encodes functional proteins. J Bacteriol 1996, 178:3544-3549.

20. Filloux A: Secretion signal and protein targeting in bacteria: a biological puzzle. J Bacteriol 2010, 192:3847-3849.

21. Ausubel FM, Brent R, Kingston RE, Moore DD, Seidman JG, Smith JA, Struhl K: Short protocols in molecular biology. 5th edition. New York: Wiley; 2002

22. Neidhardt FC, Bloch PL, Smith DF: Culture medium for enterobacteria. J Bacteriol 1974, 119:736-747.

23. Thomason L, Court DL, Bubunenko M, Costantino N, Wilson H, Datta S, Oppenheim A: Recombineering: genetic engineering in bacteria using homologous recombination. Curr Protoc Mol Biol 2007, Chapter 1 (Unit 1):1.16.1-1.16.24.

24. Baba T, Ara T, Hasegawa M, Takai Y, Okumura Y, Baba M, Datsenko KA, Tomita M, Wanner BL, Mori H: Construction of Escherichia coli K-12 in-frame, single-gene knockout mutants: the Keio collection. Mol Syst Biol 2006, 2:2006.0008

25. Haft RJ, Palacios G, Nguyen T, Mally M, Gachelet EG, Zechner EL, Traxler B: General mutagenesis of $F$ plasmid Tral reveals its role in conjugative regulation. J Bacteriol 2006, 188:6346-6353.

26. Amann E, Ochs B, Abel KJ: Tightly regulated tac promoter vectors useful for the expression of unfused and fused proteins in Escherichia coli. Gene 1988, 69:301-315.

27. Haft RJF, Gardner JG, Keating DH: Quantitative colorimetric measurement of cellulose degradation under microbial culture conditions. Appl Microbiol Biotechnol 2012, 94:223-229.

28. Collmer A, Ried JL, Mount MS: Assay methods for pectic enzymes. Methods Enzymol 1988, 161:329-335.

doi:10.1186/1471-2180-13-130

Cite this article as: DeCanio et al:: The non-pathogenic Escherichia coli strain W secretes SsIE via the virulence-associated type II secretion system beta. BMC Microbiology 2013 13:130.

\section{Submit your next manuscript to BioMed Central and take full advantage of:}

- Convenient online submission

- Thorough peer review

- No space constraints or color figure charges

- Immediate publication on acceptance

- Inclusion in PubMed, CAS, Scopus and Google Scholar

- Research which is freely available for redistribution 